\title{
Erratum: General Non-Markovian Dynamics of Open Quantum Systems [Phys. Rev. Lett. 109, 170402 (2012)]
}

Wei-Min Zhang, Ping-Yuan Lo, Heng-Na Xiong, Matisse Wei-Yuan Tu, and Franco Nori (Received 17 January 2017; published 3 February 2017)

DOI: 10.1103/PhysRevLett.118.059902

We recently found two typos in the Letter: 1. The signs of the last two terms in Eq. (2) should be exchanged, i.e., " $-a_{i}^{\dagger} a_{j} \rho(t) \mp \rho(t) a_{j} a_{i}^{\dagger}$ " should be “ $\mp a_{i}^{\dagger} a_{j} \rho(t)-\rho(t) a_{j} a_{i}^{\dagger}$," as were originally given in Refs. [19, 20] in the paper. 2. In the second line after Eq. (13), the minus sign in the equation should be a plus sign, i.e., " $\omega^{\prime}=\varepsilon_{s}-\Sigma\left(\omega^{\prime}\right)$ " should be “ $\omega^{\prime}=\varepsilon_{s}+\Sigma\left(\omega^{\prime}\right)$." No results in the Letter are affected by these typos. 